\title{
Probing the Dynamics of Phase Transformation in Nanostructures by STEM Imaging and Spectroscopy
}

\author{
Wenpei Gao ${ }^{1}$, Peter Tieu ${ }^{2}$ and Xiaoqing Pan $^{3}$ \\ ${ }^{1}$ North Carolina State University, United States, ${ }^{2}$ University of California, Irvine, California, United States, \\ ${ }^{3}$ Department of Physics and Astronomy, University of California, Irvine, CA 92697, Irvine, California, United \\ States
}

The phase transformation is commonly used to design functional materials with desirable structure and properties. The physics and chemistry at the atomic scale guide the transformation of these materials. In situ scanning/transmission electron microscopy (S/TEM) is suitable to gain insight into the transformation process at atomic resolution by applying a variety of stimulus (heating, biasing, cooling, strain, etc.) in combination with advanced techniques such as STEM diffraction imaging (4D STEM) and electron energy-loss spectroscopy (EELS). In addition, the electron beam within the microscope can be well controlled to emulate a reducing environment, inducing phase transformations as well. In this talk, we present our TEM studies of electron beam sensitive materials to demonstrate the capability of advanced S/TEM for probing the dynamics of phase transformation and chemical bond change: (1) dynamics of electron beam induced formation of nanoparticles from a precursor captured at low electron dose with aberration-corrected TEM; (2) thermally induced bond cleavage and formation within a metal-organic framework captured by vibrational electron energy-loss spectroscopy (EELS) in combination with in situ TEM imaging.

We employed in situ aberration-corrected TEM with a well-controlled electron beam to observe the transformation of $\mathrm{K}_{2} \mathrm{PtCl}_{4}$ precursor to $\mathrm{Pt}$ nanoclusters. The pristine structure of the $\mathrm{K}_{2} \mathrm{PtCl}_{4}$ was revealed at sub- $\AA$ resolution with all $\mathrm{K}, \mathrm{Pt}$, and $\mathrm{Cl}$ atoms resolved. The in situ experiment revealed a rapid transformation from the precursor to the nanocluster even under low electron dose (Figure 1A, B). Radial integration of the fast Fourier transformation (FFT) of the sequential TEM image series reflect the radial distribution function and correspond to atomic pairs with certain bond lengths, allowing for identification of the sequence of bond cleavage and cluster formation (Figure 1C). The analysis of the FFT reveal a stepwise mechanism wherein the $\mathrm{K}_{2} \mathrm{PtCl}_{4}$ precursor first decomposes into $\mathrm{K}^{+}$and $\mathrm{PtCl}_{4}{ }^{2-}$ owing to the weaker ionic bonding. The $\mathrm{PtCl}_{4}{ }^{2-}$ then decomposes into $\mathrm{PtCl}_{2}$ and $\mathrm{Cl}^{-}$. This second stage has not been reported, but is revealed here from the FFT of the high resolution images acquired under a low dose electron beam. Removal of some $\mathrm{K}$ species causes the lattice to become unstable and compounds such as $\mathrm{PtCl}_{2}, \mathrm{KCl}$, and $\mathrm{Cl}_{2}$ move about. Finally, $\mathrm{PtCl}_{2}$ is reduced to zero-valent $\mathrm{Pt}$ and the formation of the Pt nanocluster either nucleate or migrate along the surface to add to other nuclei. Our findings demonstrate a staged reduction and nucleation of Pt following the order of chemical and bonding energy.[1]

Sequential bond cleavage and formation was also observed during in situ heating of a zirconium-based metalorganic framework. Individual FFTs of an image series at increasing temperatures were integrated radially, revealing the inter-zirconium distance decreased at increasing temperatures. Vibrational EELS was performed at $60 \mathrm{kV}$ using a monochromated, spherical aberration-corrected Nion UltraSTEM with an energy resolution of $5.7 \mathrm{meV}$ to investigate the mechanisms of the lattice contraction. We observed the formation of disulfide bonds at $250{ }^{\circ} \mathrm{C}$. At higher temperatures, the weak disulfide bond was cleaved and decarboxylation occurred, leading to a second stage of contraction in the lattice. The in situ vibrational EELS results were corroborated with ex situ X-ray photoelectron spectroscopy. This sequential transformation was shown to occur in step and at varying temperatures through a combination of TEM imaging and vibrational EELS.

In situ studies of materials transformation using advanced TEM techniques can reveal details of a transformation process previously not observed. By combining the atomic resolution imaging afforded by aberration-corrected TEM with other tools, a direct understanding of structure changes can be correlated with chemical changes, giving insight into the mechanism which other material systems can be transformed. 
A
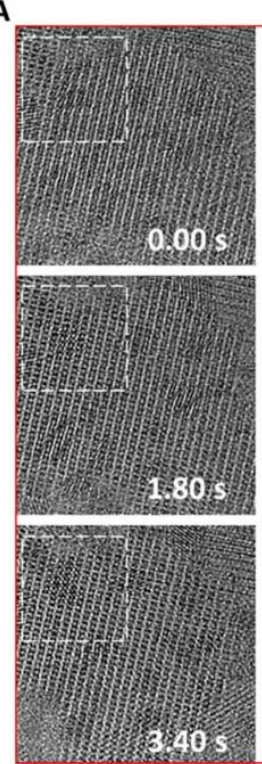
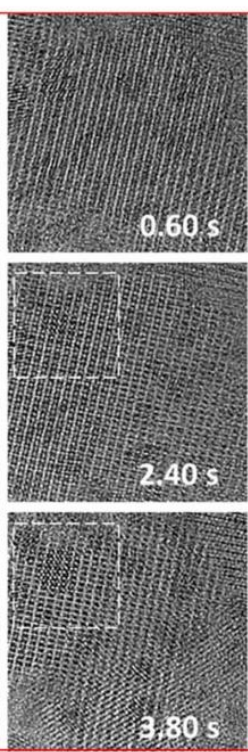

$5 \mathrm{~nm}$
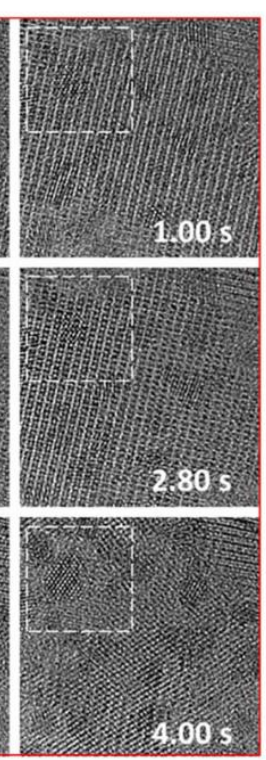

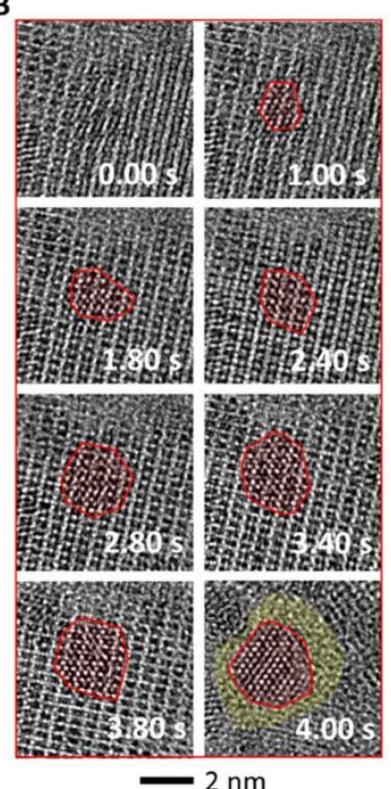

C

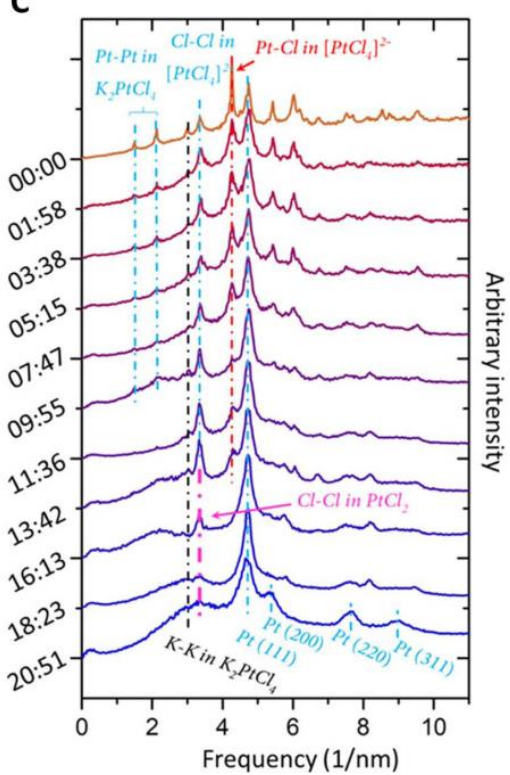

Figure 1. (A) Sequential TEM images showing the evolution of K2PtCl4 into Pt nanoclusters. The Pt nucleation process is showing in the zoomed-in images in (B) from the white dashed box in (A). The red contour lines show the edge of the newly formed Pt cluster. The yellow region highlights areas void of lattice after Pt cluster formation. (C) Radially integrated FFTs from an extended image series showing the sequential bond cleavage and formation during the transformation process.

A

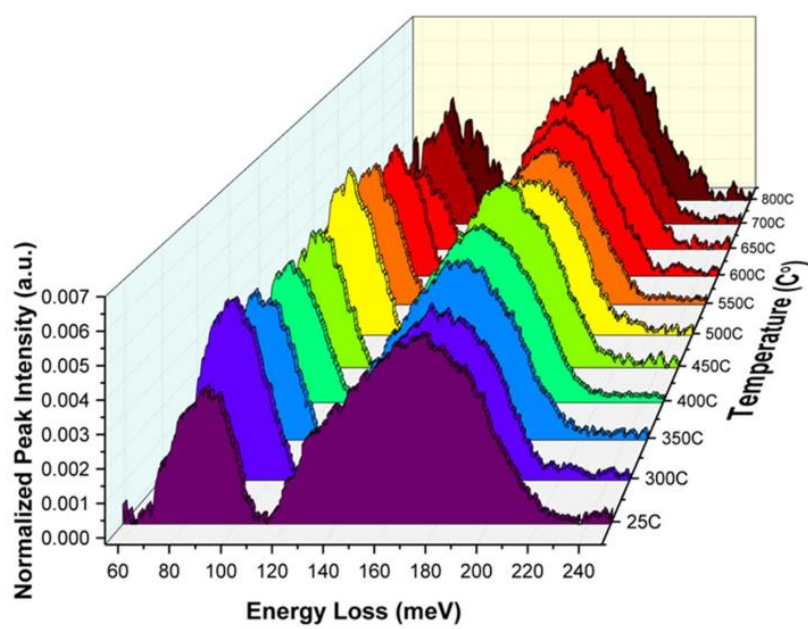

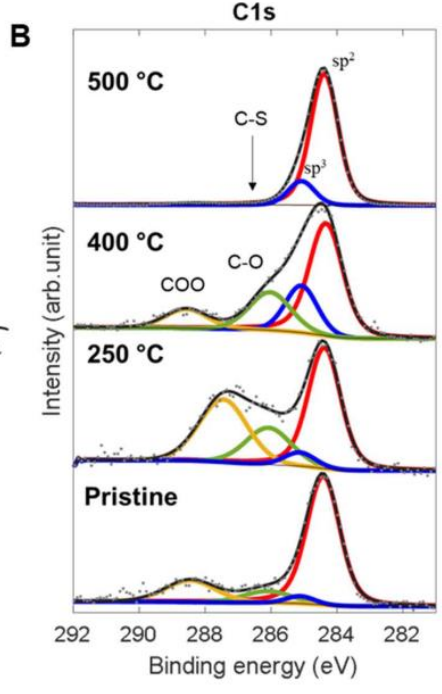

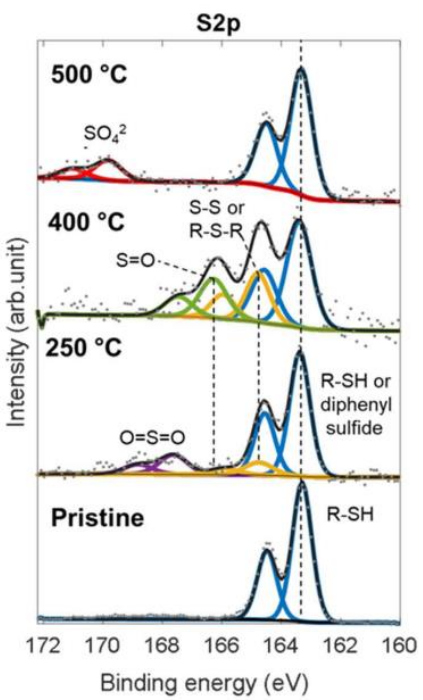

Figure 2. (A) Vibrational EELS signal acquired at increasing temperatures. The peak from $60-110 \mathrm{meV}$ is attributed to the C-S and S-S bonds. The peak from $115-225 \mathrm{meV}$ is attributed to the aromatic C-C and carboxyl bonds. (B) Ex situ X-ray photoelectron spectroscopy of $\mathrm{C} 1 \mathrm{~s}$ and $\mathrm{S} 2 \mathrm{p}$ peaks at various temperatures corroborating the formation and cleavage of the disulfide bond and the decarboxylation process.

\section{References}


[1] W. Gao, et al. Sci. Adv. 2019, 5, eaau9590

[2] This work was supported by the NSF awards under grant No. CBET-2031494 and CHE-1955786. The authors acknowledge the use of facilities and instrumentation at the UC Irvine Materials Research Institute (IMRI) supported in part by the NSF through the MRSEC program (DMR-2011967). 